\title{
A novel bioelectronic glucose sensor to process distinct electrical activities of pancreatic beta-cells *
}

\author{
Quang Vinh Nguyen, Anton Caro, Matthieu Raoux, Adam Quotb, Jean-Baptiste Floderer, \\ Yannick Bornat, Sylvie Renaud, Member, IEEE, and Jochen Lang, Member, IEEE
}

Abstract - Glucose sensors have considerably improved and facilitated therapy for type 1 diabetes. Pancreatic $\beta$-cells have been shaped during evolution as biological glucose sensors and should integrate a number of physiological relevant signals. Moreover, a biosensor based on these cells may serve for their non-invasive and long-term characterization, for drug research, tissue engineering and pre-transplantation quality control. $\beta$-cells alter their electrical activity upon exposure to glucose and physiological hormones. We have used these properties to design a biosensor. To this end extracellular signals were recorded from these cells on multi-electrode arrays and we describe here the presence of slow and rapid oscillations, both modulated by glucose. Especially slow oscillations are very robust and have an excellent signal/noise ratio. Signal processing functions were designed to separate the two activities to extract and analyze relevant parameters. These parameters correlate very well with either increasing or decreasing glucose concentrations. An electronic device is under construction, based on an embedded FPGA capable of processing multiple channels in parallel. This device shall be used as a portable and real-time biosensor regulating insulin delivery from a pump.

\section{INTRODUCTION}

Type I diabetes is a chronic disease of increasing incidence [1] with loss of pancreatic $\beta$-cells, that normally produce and secrete insulin in response to nutrients such as glucose. Therapy implies determination of blood glucose levels and corresponding injections of the potent hormone insulin. More recently transplantation of islets has been established in the case of severe and difficult to control type I diabetes. Considerable hope is placed in differentiation of stem cells though successful protocols are still not available [2].

The introduction of electrochemical glucose sensors has presented a major breakthrough in diabetes therapy [3]. The continuous monitoring of glucose levels and injection of the corresponding amounts of insulin thus became feasible. However, this approach captures only part of the information the organism uses for physiological regulation of

*Research supported by the ANR EMERGENCE "HY-BIOPACS" and funds from the Aquitaine Regional Government/FEDER to SR and JL.

Authors Q.V.N., A.Q., J.B.F., Y.B. and S.R. are with the University of Bordeaux 1, UMR CNRS 5218, Talence, FR-33405, France; authors A.C., M.R. and J.L. are with the University of Bordeaux 1, UMR CNRS 5248, Pessac, FR-33600, France (corresponding author phone: 0+33 633 755776; e-mail: j.lang@cbmn.u-bordeaux.fr). homeostasis. Within the body a group of specialized cells, the so-called $\alpha$-, $\beta$ - and $\delta$-cells, are arranged in a micro-organ to sense the nutrient levels and demand in the hormone insulin. Biological sensors, such as pancreatic islets, have been shaped during half a billion years of evolution and in contrast to currently available medical devices, they rely on a number of different relevant signals, not only glucose but also several hormones and nutrients other than glucose [4].

The integration of information about the presence of these molecules by pancreatic $\beta$-cells subsequently provides a precisely tuned signal to regulate cellular actions, such as the release of the potent hormone insulin into the bloodstream. Islet cells, including $\beta$-cells, are excitable cells similar as nerve cells. They relate signals by changing their electrical properties and this represents the first signal integration step in these cells. Therefore the electrical properties relate precisely the information obtained through different signals and reflects the activity of these cells and the demand of the

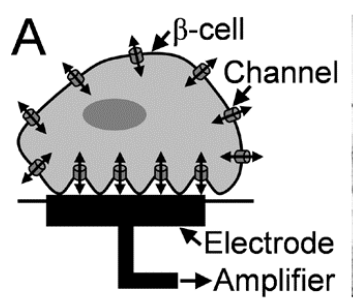

C

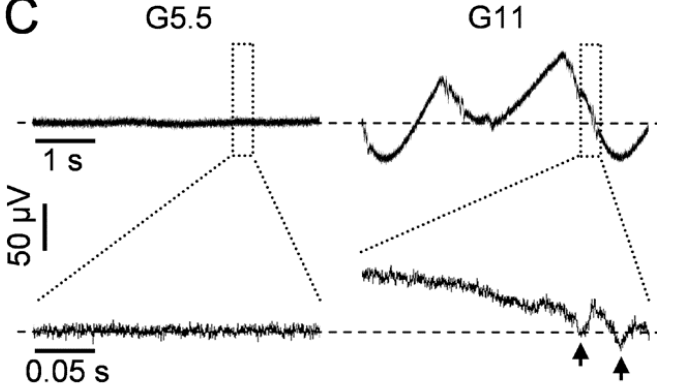

$54 \mathrm{pt}$ 0.75 in $19.1 \mathrm{~mm}$
Fig. 1: Extracellular recording of electrical signals from mouse islet cells cultured on multi-electrode arrays (MEA). A, Scheme of electrical signals generated by the activity of ion channels in a $\beta$-cell cultured on a microelectrode connected to an amplifier. B, 2-days-old culture of mouse islet cells growing on the MEA titanium nitride electrodes (planar, $30 \mu \mathrm{m}$ diameter; scale bar: $100 \mu \mathrm{m})$. C, representative electrophysiological recordings obtained from one electrode of the MEA in B. The null-voltage is indicated by horizontal broken lines. At low glucose concentration (5.5 $\mathrm{mM}, \mathrm{G} 5.5$ ) electrical oscillations are absent (left), whereas a concentration mimicking hyperglycaemia (11 mM, G11) induced low frequency oscillations (right, upper trace) on which rapid signals (action potentials) are grafted as can be seen at higher temporal resolution (see arrows at right, lower trace). 
body for insulin [5]. Electrical signals can be captured by extracellular electrodes and the corresponding devices are easy to miniaturize and do not produce heat, considerations that are important in the case of implantation [6].

A device extracting information on the activity of betacells may serve as a novel sensor for the demand in insulin and regulate an insulin infusion pump in patients suffering from type 1 diabetes. Such a device may also bypass the shortcoming of currently used sensors, i.e. their incapacity to work in a closed-loop configuration and the ensuing requirement of complex algorithms and user interventions [7]. Moreover, we propose that such a device may also serve rapidly to monitor the activity of islet-cells prior to their transplantation, in the field of tissue engineering to follow the differentiation of stem-cells into islet cells in a nondestructive manner or to be used in drug screening.

We have previously shown the general feasibility of this approach that is capturing the electrical signals by the use of extracellular electrodes and extracting the relevant information on-line which reflects the ambient levels of glucose and hormones $[8,9]$. However, the electrical signals are of small amplitude and have a low signal-to-noise ratio. Thus information extracted from a single processing algorithm presents a limited reliability. The use of the device beyond research and in a medical setting requires high reliability in the characterization process to ensure the patient security. We propose here an enhanced process based on the parallel processing of the electrical signals in two different frequency bands.

\section{A. Cell culture}

All experimental procedures reported here were in accordance with Bordeaux University policies governing the ethical use of mice for experimentation and the French Ministry of Agriculture and Forestry concerning animal care. Twelve-week-old mice were killed by cervical dislocation and their pancreata were removed and digested with collagenase NB 8 (1.1 PZ-U/ml, Serva Electrophoresis, Heidelberg, Germany). Pancreatic islets were then isolated from exocrine cells by hand-picking under a stereomicroscope (Stemi 2000, Zeiss, Thornwood, NY) and shortly trypsinised (Invitrogen, Carlsbad, CA). Cells from 80-150 islets were then seeded on the centre microelectrode arrays (60MEA200/30iR-Ti-gr, Multichannel Systems, Reuttlingen, Germany) containing 60 circular and planar electrodes in titanium nitride $(30 \mu \mathrm{m}$ diameter. To optimize the attachment of the cells, multielectrode array (MEA) surfaces were treated before seeding with a plasma air and coated for 1-2h with 50-fold diluted Matrigel (growth factor reduced, BD Biosciences, San Diego, CA, USA). After 2 days of culture in $11 \mathrm{mM}$ of glucose, extracellular recordings with MEAs were performed at $37^{\circ} \mathrm{C}$ in a continuously perifused $(0.5-1 \mathrm{ml} / \mathrm{min})$ physiological buffer consisting of (in $\mathrm{mM}$ ): $\mathrm{NaCl} 135, \mathrm{KCl} 4.8, \mathrm{MgCl} 21.2, \mathrm{CaCl} 2$ 1.2, Hepes 10 , and glucose 5.5, 8.2, 11 and $15 \mathrm{pH} 7.4$ adjusted with $\mathrm{NaOH}$ ). Each glucose concentration was applied for $20 \mathrm{~min}$.

\section{B. Data acquisition and processing}

During the whole experiment, electrical activities generated on each electrode were continuously amplified (gain: 1100) and analogically filtered at $0.1-3000 \mathrm{~Hz}$ with a MEA1060-Inv-BC-Standard system (Multichannel Systems). Analog signals were sampled at $10 \mathrm{kHz} / 12$ bits with MC_Rack acquisition hardware and software (Multichannel Systems) and stored on a personal computer for further analysis.

For the results presented in this paper, we used Matlab software to compute data. As illustrated in figure 2A, two components of the signals are processed in parallel: rapid oscillations or action potential-like signals (APs) which are of relatively high frequency $(>3 \mathrm{~Hz})$; slow oscillations or slow waves (SW) that are of low frequency and higher amplitude $(100 \mu \mathrm{V})$. Both signals are modulated by glucose and hormones but APs are generated by single cells whereas SWs are generated by intercellular coupling. Thus SWs provide important and very robust signal with an excellent signal/noise ration.

We have therefore attempted to fully exploit these signals as depicted in Fig. 2A for the data processing flow:

- signals at the output of the MC_Rack are 12-bit signed numbers, covering the signal range $[-200 \mu \mathrm{V} ; 200 \mu \mathrm{V}]$, sampled at $10 \mathrm{kHz}$.

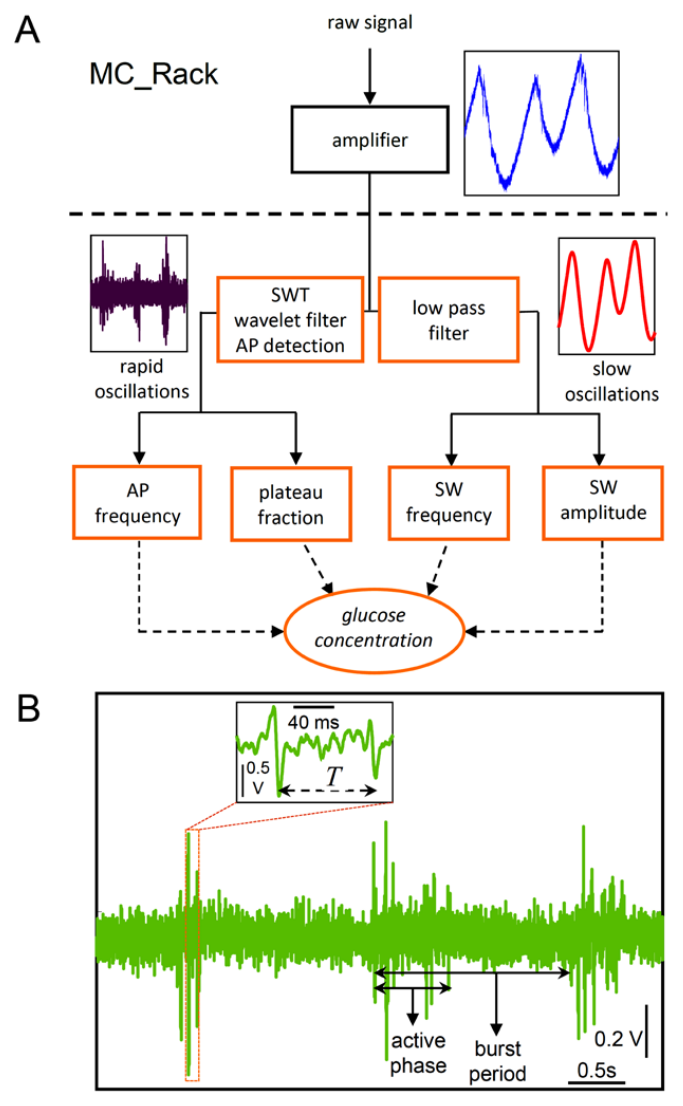

Fig. 2. Processing of signals from pancreatic ß-cells . A: Data processing flow for analysis of slow and rapid oscillations. B: Example of rapid oscillations signal and related burst features after pre-amplification (1100x): burst period is the time elapsed between 2 active phases, where an active phase corresponds to at least 2 spikes separated by $T$ ( $T$ is in [5ms; 500ms]. 
- for AP-based processing, the amplified raw signal is filtered using a wavelet filter to facilitate AP detection $[10,11]$.

We use Stationary Wavelet Transform (SWT), with a Haar mother wavelet; APs detection through an adaptive threshold, which is continuously updated and equals $2 \times \sigma(\sigma$ is the standard deviation), is performed on the $6^{\text {th }}$ level of wavelet decomposition. The detection is implemented with negative peaks of the AP signals. A resting period T of a minimum of $5 \mathrm{~ms}$ and a maximum of $0.5 \mathrm{~s}$ has to be respected between two spikes (see Fig. 2B). We subsequently compute the APs average frequency as a measure of the global activity, and the AP plateau fraction (ratio active phase/ burst period as defined in the Fig. 2B), as the fraction of time when APs occur in a burst. Indeed, it is well known that trains of rapid oscillations are interspersed by periods of silence, thus creating burst patterns. For this application, we have defined as bursts the presence of at least two directly consecutive spikes.

- for SW-based processing, the amplified raw signal is extracted by using a low-pass Butterworth filter (order of 2) with a cut-off frequency of $1 \mathrm{~Hz}$. Next the following parameters are processed: frequency and amplitude of the slow oscillations. SW frequency is defined by the time between two consecutive positive peaks, while amplitude is the difference between maximum and minimum peak values during the downward phase of one oscillation.

\section{RESULTS}

Primary $\beta$-cells were cultured on micro-electrode arrays due to changes in ion fluxes near or at the cell membrane (see Fig. 1A and B). Perifusion of these cells with low glucose (5.5 mM), a concentration that mimics normoglycemia, did not provoke any changes in the electrical signal (Fig. $1 \mathrm{C}$ ). However, when glucose was increased to higher levels, i.e. $11 \mathrm{mM}$, a concentration mimicking hyperglycemia, two distinct electrical phenomena were observed. First, large oscillations of low frequency appeared with an amplitude of 100 to $150 \mu \mathrm{V}$. Second, on top of these slow oscillations we observed rapid, short-lived oscillations of much smaller amplitude (10 to $30 \mu \mathrm{V}$; see Fig. 1C). These rapid spikes correspond to action potentials which we had demonstrated previously and described in detail their on-line analysis in real time [9].

As glucose alters the physiological activity of $\beta$-cells, we have cultured these cells on MEAs and superfused them with glucose containing buffers (Fig. 3A). Starting at $3 \mathrm{mM}$ glucose, a concentration mimicking hypoglycemia, we gradually increased the level of the sugar every 20 minutes first to 5.5 (normoglycemia) and to hyperglycemic values $(8.2,11$ and $15 \mathrm{mM})$. Subsequently glucose levels were reduced stepwise to $11,8.2,5.5$ and finally $3 \mathrm{mM}$.

First, neither slow nor rapid oscillations were observed at 3 or $5.5 \mathrm{mM}$ glucose (Fig. $3 \mathrm{~B}$ and $\mathrm{C}$, left panels). Second, we observed a very steep increase in frequencies between 5.5, 8.2 and $11 \mathrm{mM}$ glucose and a less pronounced one between 11 and $15 \mathrm{mM}$ glucose. In contrast, the amplitude of slow waves represented an all or none response with a cut-off between 5.5 and $8.2 \mathrm{mM}$ of the sugar reflecting the absence

or presence of oscillations (Fig. 3B, right panel). Similar to the changes in frequencies, the plateau phase for rapid oscillations increased in parallel to increases in glucose levels (Fig. 3C, right panel). Decreasing the glucose concentration was well reflected by a decrease in frequencies for both, rapid and slow oscillations. In contrast, there was some delay in the decrease when measuring the plateau fraction.

\section{DISCUSSION}

Our previous work $[6,8,9]$ and the current report clearly demonstrate the usefulness of pancreatic $\beta$-cells as biosensors using microelectrodes and recording of their electrical activity. As this approach has initially been pioneered for the use on neurons and heart muscle cells, which express large action potentials, the application to endocrine cells could not have been taken for granted.

We present now a data processing flow based on signal separation in two distinct frequency bands. Simple frequency and amplitude features are extracted in each band, and testify for changes in glucose concentrations. The aim of the parallel processing is to reinforce the result confidence, as the signal in each band is potentially biased by the intrinsic biological

\section{A PERIFUSION SCHEME}

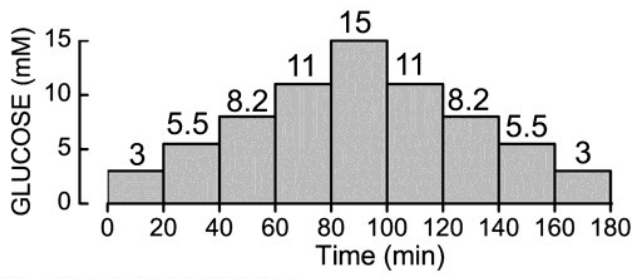

B SLOW OSCILLATIONS
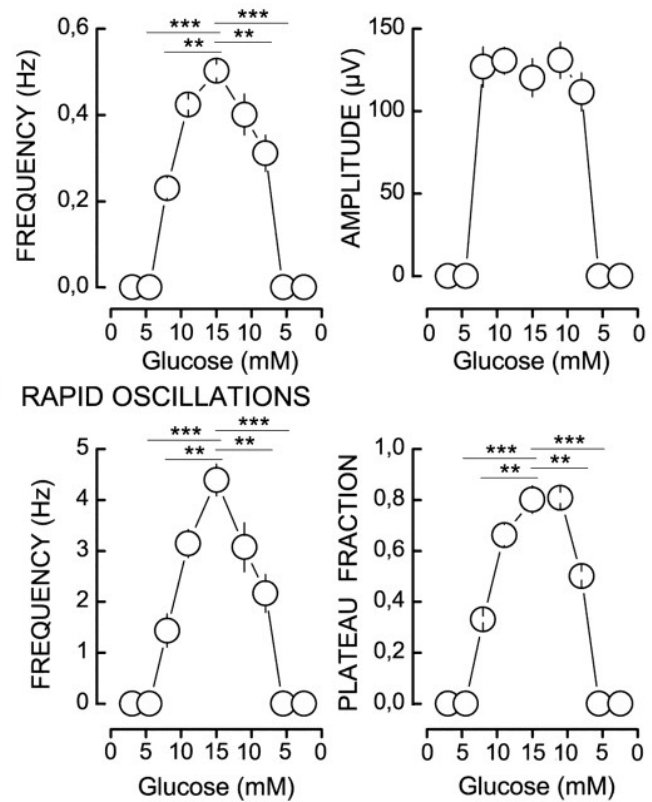

Fig. 3. Slow and rapid oscillations of field potentials in primary islet cells reflect changes in ambient glucose concentrations. A: Scheme of experiments. Islet cells cultured on multi-electrode arrays were perifused first with increasing and subsequently with decreasing glucose concentrations (each step lasted $20 \mathrm{~min}$ ). B: Results for amplitude and frequency of slow oscillations. C: Results for frequency and plateau fraction of rapid oscillations (action potentials). $\mathrm{N}=7 ;{ }^{* *}, \mathrm{p}<0.02, * * *, \mathrm{p}<0.01$ (Tukey post-hoc test). 

in this paper were conducted off-line, processing functions were specified and dimensioned for future on-chip embedding for on-line processing of multiple signals from a single MEA.

Fig. 4: Recording and processing board with an FPGA. Connector on the right is wired for recordings to the MEA and its preamplifier. Raw and processed data are stored on SDRAM cards. Analysis outputs are available on a USB port for display or post-processing.

We have therefore designed a dedicated recording and processing board (see Fig.4) with an FPGA from Xilinx ${ }^{\mathrm{TM}}$ (Spartan 3E XC3S500E) as computational core. Using this system, we have demonstrated on-line and real-time wavelet filtering and AP detection on 60 parallel channels $[6,8,9]$. Those functions were identified to have the maximum computational cost in the data processing flow given in Fig. $2 \mathrm{~A}$. We are now in the process of embedding the remaining functions, i.e. mainly SW processing, on the FPGA.

Our observation on the glucose-dependency of slow and rapid oscillations reveals two important points. First, signals were absent in at glucose concentrations below 8.2. Note that and man, were the cut-off was at about $6 \mathrm{mM}$, a concentration not tested here [12]. Second, we observed rapid reduction in activity upon reduction of glucose concentration. heterogeneity of the preparation. Although analyses presented

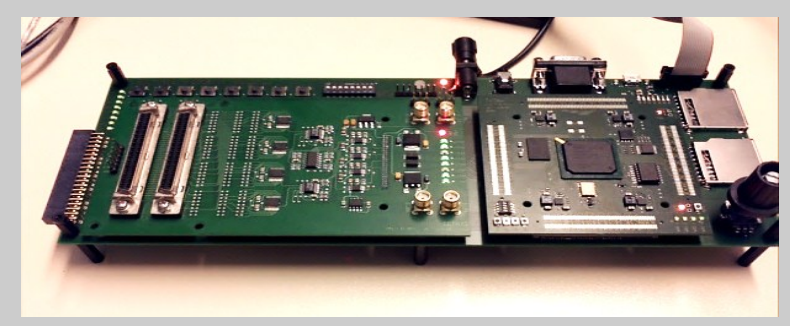

activity as it was the only stable parameter between 8.2 and $15 \mathrm{mM}$ glucose.

\section{CONCLUSION}

We have observed and extracted two different electrical signals that depend on glucose concentration in pancreatic $\beta$ cells. This shall permit us the construct a valuable algorithm for the regulation of an insulin-delivering pump in the treatment of type I diabetes. A demonstration of the first steps of this algorithm is presented here using off-line computation. An electronic device is under construction, based on an embedded FPGA capable of processing multiple channels in parallel. This device shall be used as a portable and real-time biosensor to regulate insulin delivery from a pump.

\section{ACKNOWLEDGMENT}

We thank J. Gaitan for excellent technical support.

\section{REFERENCES}

[1] D. Dabelea, "The accelerating epidemic of childhood diabetes," Lancet, vol. 373, pp. 1999-2000, 2009.

[2] J. C. Belmonte, J. Ellis, K. Hochedlinger et al., "Induced pluripotent stem cells and reprogramming: seeing the science through the hype," Nat Rev Genet, vol. 10, no. 12, pp. 878-83, 2009.

[3] T. Battelino, I. Conget, B. Olsen et al., "The use and efficacy of continuous glucose monitoring in type 1 diabetes treated with insulin pump therapy: a randomised controlled trial," Diabetologia, vol. 55, pp. 3155-3162, 2012. These properties document that culture on electrodes does not impair the physiology of the biological substrate, the islets. Moreover, these characteristics will facilitate the use as a biosensor as a steep increase in activity was only observed in the physiological relevant range of glucose concentrations. Note that concentrations such as $15 \mathrm{mM}$ or even higher are often used in-vitro but do not occur in live animals or man [5]. The fact that electrical activity increases only modestly between 11 and $15 \mathrm{mM}$ (as compared to the difference between 8.2 and $11 \mathrm{mM}$ ) is therefore not a disadvantage.

As insulin is a very potent hormone and overdosage may induce life-threatening hypoglycemia, the rapid reduction in electrical activity when reducing glucose is of considerable importance. In fact, our data suggest that the classical approach using the plateau fraction may not be the best parameter to regulate insulin delivery, for example by a pump as the return is slower. Moreover, it is much straighter forward to extract frequencies of slow and rapid oscillations than to calculate plateau fractions over an extended time period. The encoding of glucose concentration by frequency shall therefore favor implementation of online real-time data treatment.

Interestingly the amplitude of the slow oscillations did not change once this signal appeared. Use of this all-or-non signal may enhance security and help to avoid hypoglycemia. In the same vein it may serve as internal control for sensor
[4] P. E. MacDonald, J. W. Joseph, and P. Rorsman, "Glucose-sensing mechanisms in pancreatic beta-cells," Philos Trans R Soc Lond B Biol Sci, vol. 360, no. 1464, pp. 2211-25, 2005.

[5] P. Rorsman, and M. Braun, "Regulation of Insulin Secretion in Human Pancreatic Islets," Annu Rev Physiol, vol 75, 2013 (epub ahead of print; DOI: 10.1146/annurev-physiol-030212-183754).

[6] M. Raoux, G. Bontorin, Y. Bornat et al., "Bioelectronic Sensing of Insulin Demand," Biohybrid Systems: Nerves, Interfaces, and Machines, R. Jung, ed., pp. 191-202, Berlin: Wiley - VCH, 2011.

[7] R. Hovorka, "Closed-loop insulin delivery: from bench to clinical practice," Nat Rev Endocrinol, vol. 7, pp. 385-95, Jul, 2011.

[8] Y. Bornat, M. Raoux, Y. Boutaib et al., "Detection of electrical activity of pancreatic $\beta$-cells using micro-electrode arrays." Fifth IEEE International Symposium on Electronic Design, Test and Application, 2010. DELTA '10. pp. 233-236.

[9] M. Raoux, Y. Bornat, A. Quotb et al., "Non-invasive long-term and real-time analysis of endocrine cells on micro-electrode arrays," $J$ Physiol, vol. 590, pp. 1085-91, 2012.

[10] A. Quotb, Y. Bornat, and S. Renaud, "Wavelet transform for real-time detection of action potentials in neural signals," Front Neuroeng, vol. 4, pp. 7, 2011.

[11]A. Quotb, Y. Bornat, M. Raoux et al., "NeuroBetaMed: A reconfigurable wavelet-based event detection circuit for in vitro biological signals." 2012 IEEE International Symposium on Circuits and Systems ( ISCAS), pp. 1532-1535.

[12] J. C. Henquin, D. Dufrane, and M. Nenquin, "Nutrient control of insulin secretion in isolated normal human islets," Diabetes, vol. 55, pp. 3470-7, 2006. 